\title{
Relief of branch pulmonary artery stenosis reduces pulmonary valve insufficiency in a swine model
}

\author{
Christopher J. Petit, MD, ${ }^{\text {a }}$ Matthew J. Gillespie, MD, ${ }^{\text {a }}$ Matthew A. Harris, MD, ${ }^{\text {a }}$ Travis L. Seymour, BA, ${ }^{a}$ \\ Timothy Y. Liu, BA, ${ }^{\mathrm{a}}$ Azeem Khan, MD, ${ }^{\mathrm{b}}$ J. William Gaynor, MD, ${ }^{\mathrm{b}}$ and Jonathan J. Rome, MD ${ }^{\mathrm{a}}$
}

\begin{abstract}
Objective: We sought to determine the impact of relieving branch pulmonary artery stenosis on pulmonary valve insufficiency and right ventricular function. Long-standing pulmonary insufficiency causes progressive right ventricular dilatation, leading to decreased right ventricular function. Adults with pulmonary insufficiency are at risk of decreased exercise tolerance, arrhythmias, and sudden cardiac death. Branch pulmonary artery stenosis frequently occurs in these patients, and the presence of branch stenosis may exacerbate valve insufficiency.
\end{abstract}

\begin{abstract}
Methods: Neonatal piglets $(n=7)$ underwent surgery to create pulmonary insufficiency and left pulmonary artery stenosis. At 3 months of age, the animals underwent baseline cardiac magnetic resonance imaging followed by stenting of the left pulmonary artery. A repeat magnetic resonance imaging scan was performed 1 week after intervention. Magnetic resonance imaging evaluation included (1) velocity mapping to assess the forward and reverse flow at the main, left and right pulmonary arteries, and aorta; and (2) volumetric assessment of the right ventricle.
\end{abstract}

Results: Left pulmonary artery flow increased from $14.5 \%$ to $36.3 \%$ of total net flow after stenting $(P<.01)$. Pulmonary regurgitation decreased from $38.7 \%$ to $27.4 \%(P<.02)$. Right ventricular ejection fraction improved from a median of $53.5 \%$ to $58.2 \%$ after stenting $(P<.01)$. Cardiac index improved from a median of 2.7 to $3.5 \mathrm{~L} / \mathrm{min} / \mathrm{m}^{2}(P=.01)$.

Conclusion: Relief of branch pulmonary artery stenosis reduces insufficiency and improves right ventricular systolic function in this animal model. This supports the practice of aggressive intervention in patients with branch pulmonary artery stenosis and pulmonary insufficiency.

Pulmonary insufficiency (PI) commonly occurs after surgery for many forms of congenital heart disease, most notably tetralogy of Fallot (TOF). Right ventricle (RV) volume overload secondary to long-standing PI is associated with exercise intolerance, syncope, ventricular arrhythmias, and sudden cardiac death. ${ }^{1-3}$ The degree of PI has been found to be predictive of adverse events in these patients. ${ }^{4,5}$ Therefore, there has been increased interest in earlier intervention on the RV outflow tract in adults with moderate to severe PI and RV dilation. ${ }^{6-8}$ Timely intervention, including surgical valve replacement, has been shown to reverse the deleterious effects of RV dilation, to improve symptoms, and to reduce proclivity to arrhythmia in these patients. ${ }^{9,10}$ However, the lifespan of valved conduits is limited secondary to ensuing

From the Division of Pediatric Cardiology, Department of Pediatrics, ${ }^{a}$ Division of Cardiothoracic Surgery, Department of Surgery, ${ }^{\mathrm{b}}$ Children's Hospital of Philadelphia and the University of Pennsylvania School of Medicine, Philadelphia, Pa. Disclosures: The authors have no conflicts of interest to disclose.

This study was funded by a Career Development Grant awarded to Dr Christopher Petit from The Children's Hospital of Philadelphia.

Received for publication Oct 20, 2008; revisions received Jan 12, 2009; accepted for publication Feb 16, 2009; available ahead of print April 13, 2009.

Address for reprints: Christopher J. Petit, MD, Texas Children's Hospital, Section of Cardiology, 6621 Fannin St, MC-19345 C, Houston, TX 77030 (E-mail: cjpetit@ texaschildrenshospital.org).

J Thorac Cardiovasc Surg 2009;138:382-9

$0022-5223 / \$ 36.00$

Copyright (c) 2009 by The American Association for Thoracic Surgery doi: 10.1016/j.jtcvs.2009.02.030 conduit valve insufficiency and conduit stenosis, and these patients often undergo multiple repeated operations to revise their RV outflow as a result. Because of the increased morbidity and mortality risk associated with repeat sternotomy, less-invasive interventions to alleviate PI have been sought, including percutaneous valve implantation. ${ }^{11}$ Unfortunately, only patients with an RV to pulmonary artery (PA) conduit are candidates for such an intervention currently.

Many patients with TOF have significant residual branch PA stenosis after surgical repair. ${ }^{12-14}$ These patients have reduced exercise tolerance compared with other patients with TOF. ${ }^{15}$ There has been indirect evidence that branch PA stenosis exacerbates PI. ${ }^{16}$ However, the relationship between PI and long-standing branch PA stenosis has not been directly studied. In this study, we measured the impact of relieving left pulmonary artery (LPA) stenosis on PI in a swine model of repaired TOF. Our hypothesis is that relief of unilateral branch PA stenosis alleviates PI by improving antegrade pulmonary arterial flow and enhancing overall hemodynamic efficiency.

\section{MATERIALS AND METHODS Surgery}

Neonatal Yorkshire piglets $(n=13)$ were brought to the operating room at the Laboratory Animal Facility at the Children's Hospital of Philadelphia. The animals were a median of 13 days old (11-17 days) and a median of 3.9 $\mathrm{kg}$ (2.8-6.4). The animals were placed under general endotracheal 


$$
\begin{aligned}
& \text { Abbreviations and Acronyms } \\
& \text { EDV }=\text { end-diastolic volume } \\
& \text { ESV }=\text { end-systolic volume } \\
& \text { LPA }=\text { left pulmonary artery } \\
& \text { LV }=\text { left ventricle } \\
& \text { MPA }=\text { main pulmonary artery } \\
& \text { MRI }=\text { magnetic resonance imaging } \\
& \text { PA }=\text { pulmonary artery } \\
& \text { PBF }=\text { pulmonary blood flow } \\
& \text { PI }=\text { pulmonary insufficiency } \\
& \text { RF }=\text { regurgitant fraction } \\
& \text { RPA }=\text { right pulmonary artery } \\
& \text { RV }=\text { right ventricle } \\
& \text { TOF }=\text { tetralogy of Fallot }
\end{aligned}
$$

anesthesia using $1 \%$ to $3 \%$ isoflurane. After a dose of intravenous cefazolin $(25 \mathrm{mg} / \mathrm{kg})$, a left thoracotomy was performed. After careful blunt dissection, the LPA was identified and isolated. A 2-mm segment of 4.0-mm polytetrafluoroethylene (Gore-Tex; Gore Industries, Newark, DE) shunt material was cut open and wrapped around the proximal LPA and sewn with 6.0 suture. Then a curved clamp was placed across the RV outflow tract, including the valve annulus. Care was taken to ensure that approximately one half of the outflow tract was attained within the clamp. A longitudinal incision was made across the valve annulus onto the main pulmonary artery (MPA). Pulmonary valve tissue was cut away, and a diamond-shaped polytetrafluoroethylene (Gore-Tex) transannular patch was then sewn over the opened outflow tract. After removal of the clamp, the pericardium was closed, the left lung was reexpanded, and the thoracotomy was closed in a layered fashion. After giving a local infusion of bupivacaine $(1 \%)$ and a single dose of Buprenex $(0.1 \mathrm{mg} / \mathrm{kg})$ intramuscularly, the animals were extubated and monitored closely for 48 hours for signs of distress, wound dehiscence, infection, or pain. A fentanyl transdermal patch $(25 \mu \mathrm{g})$ was placed for the first 48 postoperative hours. Two additional control animals underwent pulmonary valve resection and transannular patch as described above, but without the band on the LPA. The Institutional Animal Care and Use Committee of the Children's Hospital of Philadelphia approved this protocol.

\section{Cardiac Magnetic Resonance Imaging}

Approximately 3 months after surgery, the animals were taken for baseline cardiac magnetic resonance imaging (MRI). The baseline study (MRI 1) was immediately followed by LPA stent placement in the catheterization laboratory (see below). One week later, repeat cardiac MRI (MRI 2) was performed to assess the impact of relief of LPA stenosis. The cardiac MRI study protocol was identical for both pre- and post-intervention studies. The animals were placed under general endotracheal anesthesia and $21 \%$ oxygen, using $1 \%$ to $3 \%$ isoflurane, and then transported to the MRI scanning area. All scans were performed on a 1.5T Siemens Avanto (Siemens Corp, New York, NY) scanner. All scans were reviewed and interpreted by a single observer (M.A.H.).

\section{Phase-Contrast Magnetic Resonance}

At both baseline and follow-up MRI studies, phase contrast magnetic resonance acquisition was applied at the main and branch pulmonary arteries and at the aorta for flow quantification. On the post-LPA stent MRI, LPA phase contrast magnetic resonance data was acquired distal to the stent to avoid any stent-produced artifact. The typical imaging parameters included an echo time of $3.5 \mathrm{~ms}, 3$ segments, a temporal resolution of 40 $\mathrm{ms}$, a flip angle of 25 degrees, a matrix of $256 \times 128$, and a voxel size of
$2.0 \times 1.5 \times 5.0 \mathrm{~mm}$ (slice thickness) with a resulting signal-to-noise ratio of approximately 1.0. Four averages were used for improving the signal-tonoise ratio and mitigating respiratory artifact. The velocity limit was set initially at $150 \mathrm{~cm} / \mathrm{sec}$ and increased as necessary to accommodate increased flow velocities. Phase contrast magnetic resonance data analysis involved contouring regions of interest throughout all phases of the cardiac cycle. Forward, regurgitant, and net flows were then automatically calculated from the resulting flow-time curves. The regurgitant fraction (RF) through a region of interest is defined as follows: $\mathrm{RF}=$ (reverse flow/forward flow) $\times 100$. Fractional branch PA pulmonary blood flow $(\mathrm{PBF})$ distribution is calculated as follows: Fractional branch PA PBF $=$ (net branch PA flow/net total $\mathrm{PBF}) \times 100$. Indexed values were obtained by calculating body surface area based on an accepted conversion formula for swine: ${ }^{17}$ body surface area $($ meters squared $)=0.097 *$ weight $(\text { kilograms })^{0.656}$.

\section{Ventricular Volume Analysis}

Cine short-axis imaging of the ventricles was acquired from base to apex of the heart using 8 contiguous slices 6 to $8 \mathrm{~mm}$ thick, depending on the heart size. The RV systolic function analysis involved contouring the blood pool at end diastole and end systole at each level of the volume data set, thereby quantifying the end-diastolic volume (EDV) and end-systolic volume (ESV). The stroke volume is defined as the difference between the EDV and the ESV, and the ejection fraction is defined as the stroke volume/EDV $\times 100$.

After MRI 2, the animals were euthanized and the hearts were harvested for gross inspection. Two control animals (5 months of age) without branch PA stenosis also underwent cardiac MRI to evaluate the distribution of flow to the right and left lungs.

\section{Catheterization}

The animals were taken directly from the MRI scanner after MRI 1 to the animal catheterization laboratory in the Laboratory Animal Facility. General anesthesia was continued using $1 \%$ to $2 \%$ isoflurane. The groins were prepped and draped using sterile technique, and a short sheath (7F) was placed in the femoral vein percutaneously. Intravenous doses of cefazolin $(25 \mathrm{mg} / \mathrm{kg})$ and heparin $(100 \mathrm{U} / \mathrm{kg})$ were given once vascular access was obtained. Hemodynamic evaluation was performed, including measurement of the RV, MPA, right pulmonary artery (RPA), and distal LPA pressures. Pulmonary capillary wedge pressures were measured bilaterally. Angiography was performed at the MPA, and measurements were made of the diameters of the RPA, MPA, and the proximal and distal LPA. The short sheath was then replaced with a $9 \mathrm{~F}$ to $11 \mathrm{~F}$ sheath, which was advanced into the distal LPA. A premounted endovascular stent $(10 \times 19 \mathrm{~mm}$ or $10 \times 29 \mathrm{~mm})$ was deployed across the banded region of the LPA. Care was taken to place the stents distally within the banded region, so that follow-up MRI velocity mapping could be obtained at the proximal RPA with minimal interference from the stent. In 5 animals, the stents were post-dilated with balloons 12 to $14 \mathrm{~mm}$ in diameter to achieve a size equal to that of the distal LPA. In 1 animal, a second stent was deployed because of migration of the first stent distal to the region of narrowing. After stenting, angiography was repeated. All vascular lines were removed, and the animals were extubated and monitored closely for 48 hours. The animals received aspirin $(325 \mathrm{mg})$ daily until the day of the follow-up MRI.

\section{Statistics}

Values are presented as median (range). During many of the cardiac MRI scans, more than 1 velocity map of the pulmonary arteries was taken. In this situation, a mean value was calculated, and this mean value was used in the analysis. Velocity mapping data were internally verified using Pearson correlation comparing net forward flow at the MPA with the net flow at the ascending aorta, and with the sum of the net flow of the RPA and LPA Pearson correlation testing was also performed to ensure correlation between net flow measured across the pulmonary and aortic valves, as well 

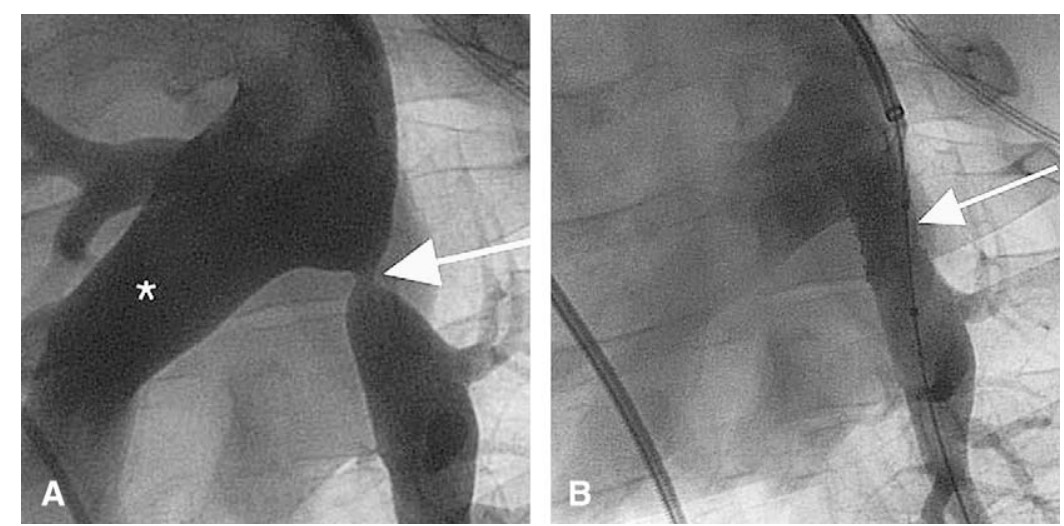

FIGURE 1. Angiography of pulmonary arteries. Angiography performed before (A) and after (B) stenting of LPA in 1 animal subject demonstrates the nature of the stenosis. Pre-stenting, there is moderate proximal stenosis (white arrow) representing the region that was banded during surgery. Distal to this band, the LPA remains smaller than the distal RPA. After stenting (B), there is complete resolution of the narrowing (white arrow). The white asterisk denotes the right pulmonary artery.

as to the RPA and LPA. Finally, correlation was performed in post-stent studies to ensure that LPA flow measured distal to the stent approximated the value calculated by subtracting RPA from MPA flow. Pre- and post-stent MRI data were compared for each animal. Continuous variables were compared using the paired Student $t$ test. Dichotomous variables were compared with chi-square and Fisher's exact tests. Statistical analysis was performed using Prism 5.0 (Graphpad Software, Inc, San Diego, CA).

\section{RESULTS}

Of the 13 animals, 10 survived the surgical procedure. Three animals survived the surgical procedure but died before completion of the protocol. Nonsurgical causes of death included noncardiac medical problems prompting euthanasia and complications surrounding general anesthesia induction. The 7 animals that completed the protocol underwent baseline MRI and catheterization at a mean of 96 (71-113) days from surgery, at a weight of $32(23-60) \mathrm{kg}$.

At the time of catheterization, the LPA at the banded region measured $3.4(2.9-4.0) \mathrm{mm}$ in diameter, compared with 11.9 (8-15.5) $\mathrm{mm}$ distally. The LPA diameter distal to the band was significantly smaller than the distal RPA diameter $(P<.01)$ (Figure 1$)$. Hemodynamic data obtained

TABLE 1. Branch pulmonary artery diameters, pressures, and flow

\begin{tabular}{|c|c|c|c|}
\hline & LPA (range) & RPA (range) & $P$ value \\
\hline Proximal diameter $(\mathrm{mm})$ & $3.4(2.9-4)$ & $19.3(16-25)$ & $<.01$ \\
\hline Distal diameter $(\mathrm{mm})$ & $13.2(8-15.5)$ & $20.7(16.5-27)$ & $<.01$ \\
\hline $\begin{array}{l}\text { Systolic pressure } \\
(\mathrm{mm} \mathrm{Hg})\end{array}$ & $15(9-22)$ & $26(19-42)$ & $<.01$ \\
\hline $\begin{array}{l}\text { Mean pressure } \\
\quad(\mathrm{mm} \mathrm{Hg})\end{array}$ & $10(6-15)$ & $16(10-28)$ & $<.01$ \\
\hline Forward flow (\%) & $14.5(3.2-20)$ & $85.5(80-96.8)$ & $<.01$ \\
\hline \multicolumn{4}{|l|}{ Post-stent } \\
\hline $\begin{array}{l}\text { Systolic pressure } \\
\text { (mm Hg) }\end{array}$ & $25(18-36)$ & $27(20-39)$ & NS \\
\hline Forward flow $(\%)$ & $36.3(11-48)$ & $63.7(52-89)$ & $<.01$ \\
\hline
\end{tabular}

$L P A$, Left pulmonary artery; $R P A$, right pulmonary artery; $N S$, not significant. during catheterization revealed a median LPA systolic pressure of 15 (9-22) $\mathrm{mm} \mathrm{Hg}$, with a median gradient to the MPA of 11 (4-22) mm Hg (Table 1). The median transpulmonary gradient on the left was $4(1-7) \mathrm{mm} \mathrm{Hg}$, compared with $9(2-16) \mathrm{mm} \mathrm{Hg}$ on the right $(P=.03)$. After stenting of the banded region of the LPA, the diameter of the proximal LPA increased to a median of $11(9-12) \mathrm{mm}(P<.01)$. There was no pressure gradient between the MPA and the LPA after stenting in each case.

Cardiac MRI velocity mapping demonstrated that at baseline, the LPA received a median of $14.5 \%(3.2-20)$ of net flow from the MPA. This improved to $36.3 \%(11-48)$ after LPA stenting $(P<.01)$ (Figure 2$)$. For the 2 control animals without LPA stenosis, forward flow to the LPA was $40 \%$ and $43 \%$ of net flow. There was strong correlation among

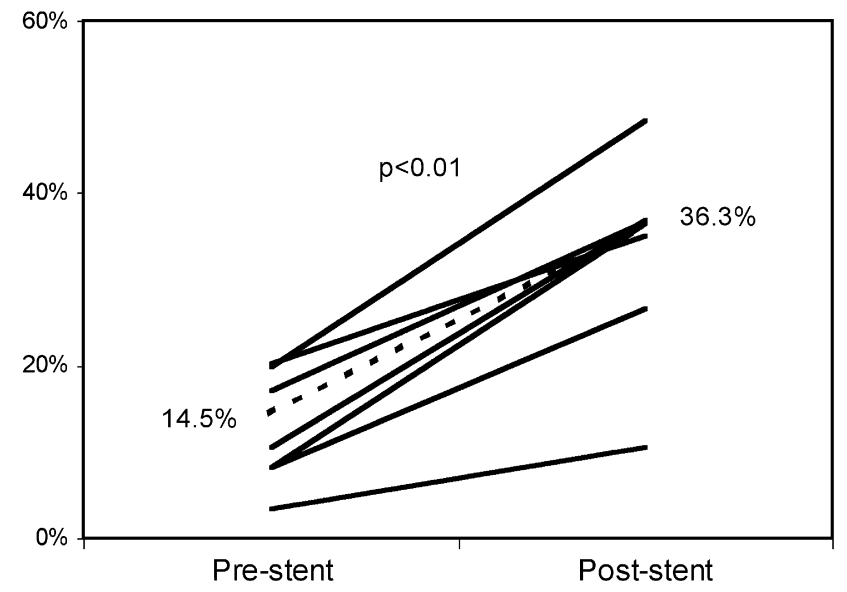

FIGURE 2. Flow to the LPA before and after stenting. Velocity mapping by cardiac MRI was used to measure flow (\%) to the branch pulmonary arteries. Net flow (calculated as total flow-retrograde flow = net flow) to the LPA at baseline was a mean (dashed line) of $14.5 \%$, which improved to $36.3 \%$ after stenting $(P<.01)$. 

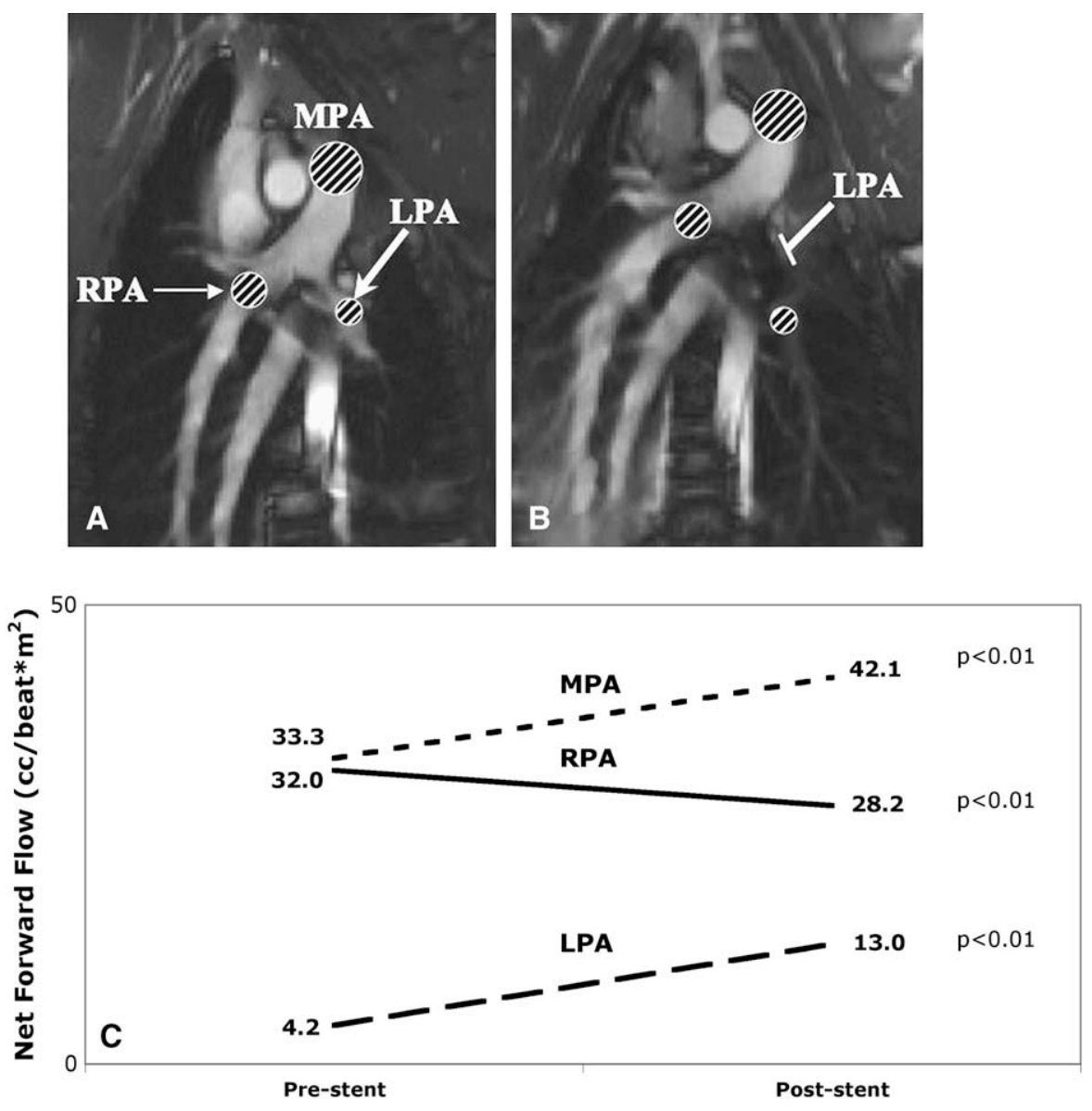

FIGURE 3. Velocity mapping of the pulmonary arteries before and after LPA stenting. Velocity mapping by MRI of the MPA, RPA, and LPA was performed before (A) and after (B) LPA stenting. To measure LPA flow after stenting, velocities were sampled distal to the region of the stent to avoid signal dropout (B). The majority of net flow pre-stent (C) was delivered from the MPA to the RPA, with little net flow to the LPA. However, post-stent, net flow to the LPA increased significantly, and RPA net flow decreased. In addition, MPA net flow increased significantly after LPA stenting. RPA, Right pulmonary artery; $M P A$, main pulmonary artery; $L P A$, left pulmonary artery.

velocity map measurements of flow among the MPA, LPA, and RPA. At baseline, the sum of net RPA and LPA flow $\left(\mathrm{RPA}_{\text {net }}+\mathrm{LPA}_{\text {net }}=\mathrm{MPA}\right.$ net $)$ correlated with net flow at the MPA $(\mathrm{R}=0.93)$ and net flow $\left(\mathrm{RPA}_{\text {net }}+\mathrm{LPA}_{\text {net }}\right.$ $=$ Aorta $\left._{\text {net }}\right)$ at the aorta $(\mathrm{R}=0.94)$. Post-stenting, the RPA and LPA net sum correlated with net flow at the MPA ( $\mathrm{R}=$ $0.93)$ and at the aorta $(\mathrm{R}=0.90)$.

Velocity mapping measurement of net flow after LPA stenting demonstrated that the net flow to the LPA increased $(P<.01)$, whereas the net flow to the RPA decreased $(P<$ $.01)$ (Figure 3). The net flow at the level of the MPA also increased $(P<.01)$. This reflected the decrease in pulmonary RF (Figure 4). The mean RF at the MPA at baseline was $38.7 \%(21.2-45.8)$ and decreased to $27.4 \%(11.3-38.8)$ after stenting of the LPA $(P=.002)$. The branch RF at the level of the proximal RPA $(P=.30)$ and LPA $(P=.28)$ did not significantly change after LPA stenting.

MRI volumetric assessment was performed on the RV before and after intervention. The RV to left ventricle
(LV) volume ratio at end diastole was 1.4 before stenting, and this did not change 1 week after LPA stenting (Figure 5). The RV EDV did not decrease significantly 1 week after LPA stenting. However, the RV ESV did decrease from a median of $44.9(35.8-58.3) \mathrm{mL} / \mathrm{m}^{2}$ to $39.3(29.4-49)$ $\mathrm{mL} / \mathrm{m}^{2}(P=.03)$ (Table 2). The RV ejection fraction improved from $53.5 \%$ to $58.2 \%(P<.01)$. Improved pump function of the RV correlated with the increase in net flow through the MPA.

Finally, cardiac index was assessed by velocity mapping at the MPA and the aorta (Figure 6). The cardiac index improved in all 7 animals, from a median of $2.7(1.7-3.4) \mathrm{L} /$ $\mathrm{min} / \mathrm{m}^{2}$ at baseline to $3.5(2.7-4.4) \mathrm{L} / \mathrm{min} / \mathrm{m}^{2}$ post-stenting $(P=.01)$. To ensure that the increase in cardiac index was not due to an increased inotropic or stressed state, heart rate was compared at time of baseline and follow-up MRI scan. Median heart rate was 93 (74-143) beats/min at baseline and $98(88-136)$ beats/min at the time of follow-up MRI scan $(P=.62)$. 

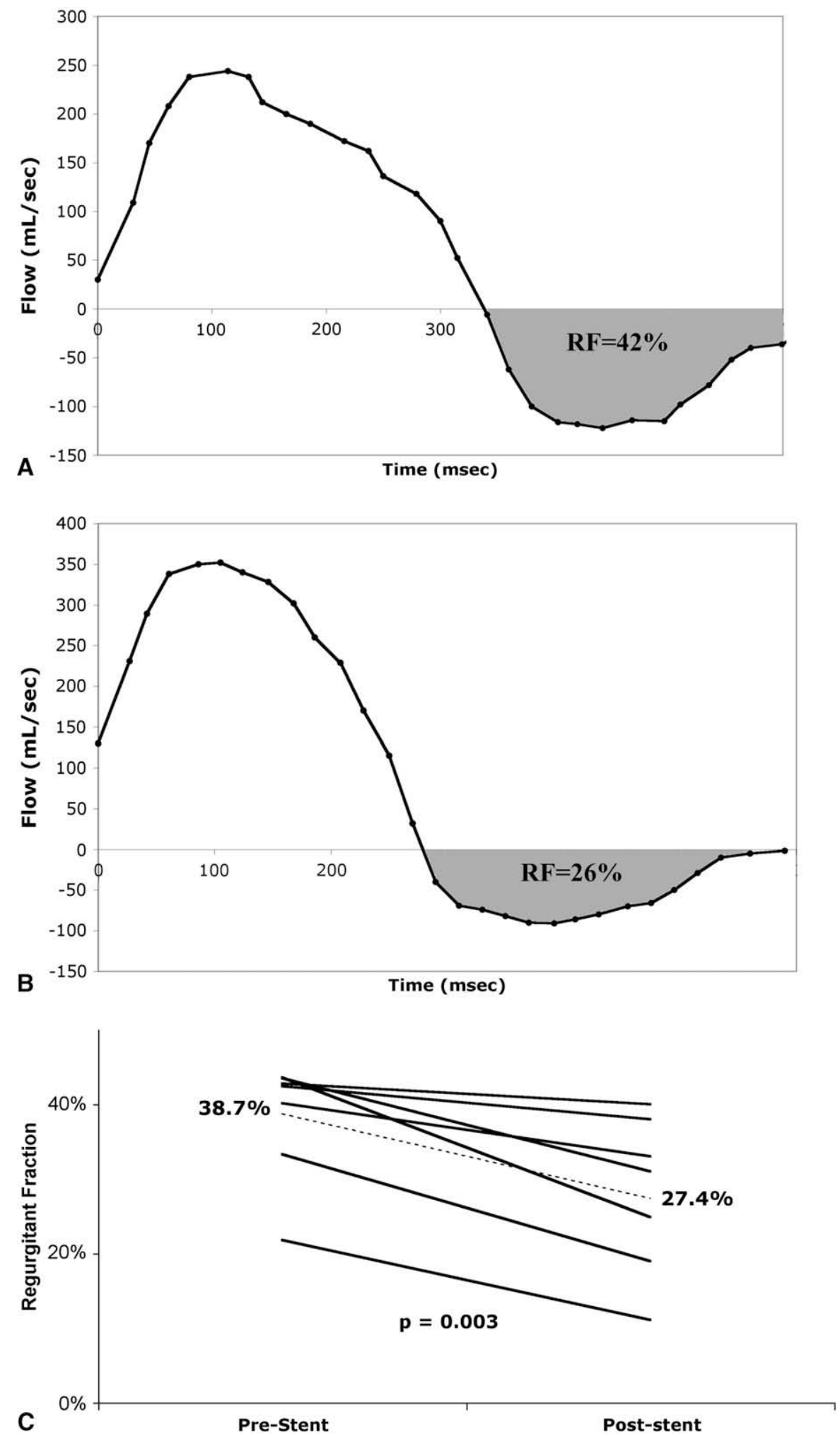

FIGURE 4. Pulmonary insufficiency before and after intervention. Pulmonary valve insufficiency was measured by velocity mapping at the MPA. Velocity mapping in one subject is shown before (A) and after (B) stenting. Forward flow is represented by the area under the curve above the baseline, and reverse flow is represented by the area under the curve below the baseline. In this subject, PI decreased after LPA stenting. For the group (C), the mean regurgitant fraction at baseline was $38.7 \%$, and this decreased to $27.4 \%$ after LPA stenting $(P=.002) . R F$, Regurgitant fraction. 


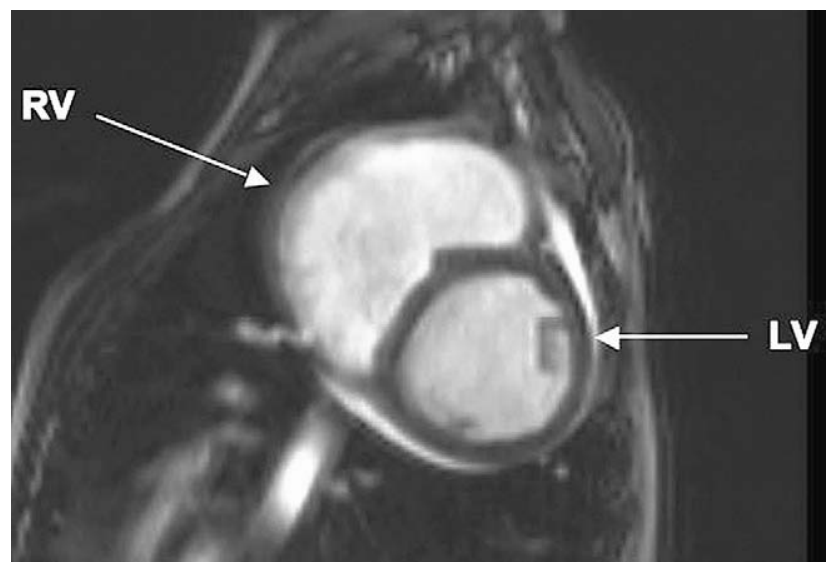

FIGURE 5. Relative volumes of the RV and LV at end diastole. Structural MRI scan of the ventricles in short axis demonstrates RV enlargement as a result of volume-loading caused by pulmonary insufficiency. Mean $\mathrm{RV}: \mathrm{LV}$ end-diastolic volume ratio was 1.4:1. $R V$, Right ventricle; $L V$, left ventricle.

\section{DISCUSSION}

Among adults with repaired TOF, there is a high prevalence of clinically significant PI. Although this can be well tolerated in the short term, long-standing PI causes RV dilatation and dysfunction. ${ }^{18}$ Common clinical practice has been to delay surgical pulmonary valve replacement until the onset of symptoms, signs of RV dilatation, or ventricular arrhythmias. This practice stems from the desire to avoid risks of repeated sternotomy, with the associated morbidities and mortality. In addition, it is recognized that implanted valves, in time, fail on the basis of restenosis and valve incompetence. Thus, even after valve replacement, many patients are left with significant PI. The timing for surgical pulmonary valve replacement remains controversial, because this patient population faces the prospect of multiple operations for pulmonary valve replacement. ${ }^{8}$

A significant number of adults and adolescents with repaired TOF have branch PA stenosis and unequal PBF, most commonly LPA stenosis with corresponding decreased left-sided perfusion, as the result of a combination of developmental anomalies and possibly as a consequence of the

TABLE 2. Volumetric analysis of the right ventricle before and after left pulmonary artery stent

\begin{tabular}{lllr}
\hline & Pre-stent (range) & Post-stent (range) & $\boldsymbol{P}$ value \\
\hline RV EDV $\left(\mathrm{mL} / \mathrm{m}^{2}\right)$ & $96.7(75.2-122.8)$ & $93.0(66.8-111.7)$ & .23 \\
RV ESV $\left(\mathrm{mL} / \mathrm{m}^{2}\right)$ & $44.9(35.8-58.3)$ & $39.3(29.4-49)$ & .01 \\
RV EF $(\%)$ & $53.5(47.9-63.4)$ & $58.2(53.5-61.7)$ & $<.01$ \\
RV EDV:LV EDV & $1.40(0.95-1.75)$ & $1.41(0.89-1.98)$ & .22 \\
Cardiac index & $2.7(1.7-3.4)$ & $3.5(2.6-4.4)$ & .01 \\
$\quad\left(\mathrm{~L} / \mathrm{min} / \mathrm{m}^{2}\right)$ & & & \\
Heart rate $($ beats/min) & $93(74-143)$ & $98(88-136)$ & .62 \\
\hline
\end{tabular}

$R V$, Right ventricle; $E D V$, end-diastolic volume; $E S V$, end-systolic volume; $E F$, ejection fraction.

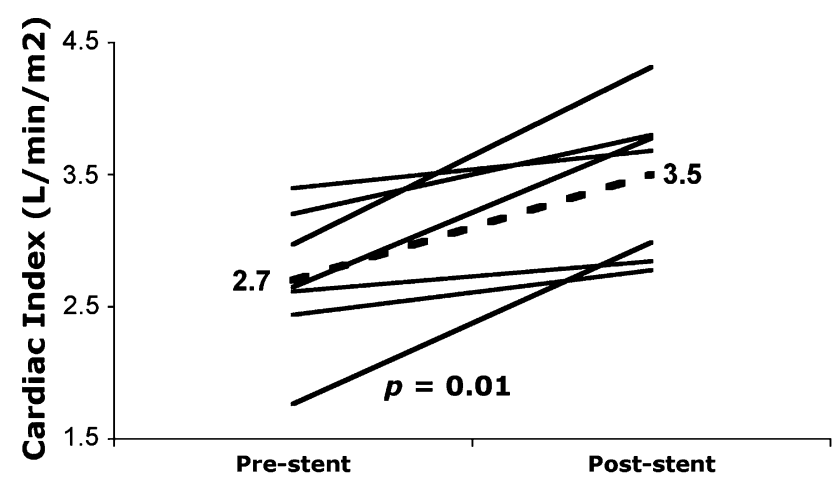

FIGURE 6. Cardiac index measured by velocity mapping. Cardiac index was measured by velocity mapping at the ascending aorta. For all animal subjects, the cardiac index improved after LPA stenting. Mean cardiac index (dashed line) improved from 2.7 to $3.5 \mathrm{~L} / \mathrm{min} / \mathrm{m}^{2}(P=.01)$.

surgical approach. ${ }^{13,14,19,20}$ It has been shown that patients with TOF and branch PA stenosis have significantly lower exercise tolerance than other patients with TOF. ${ }^{15}$ Exercise intolerance is thought to be due to the ventilation:perfusion mismatch that is seen in these patients. ${ }^{21}$ It has also been postulated that the presence of branch PA stenosis exacerbates PI. ${ }^{22,23}$ Ilbawi and colleagues ${ }^{16}$ reviewed the charts of 264 patients with TOF who had undergone catheterization after repair. They found that in the group of patients with moderate-to-severe PI and RV volume overload, both assessed by angiographic methods, branch stenosis was more common. However, the location, distribution, and severity of branch stenosis in these patients were unclear. In another study, Chaturvedi and colleagues ${ }^{24}$ simulated acute unilateral branch pulmonary atresia in patients with repaired TOF and PI. By using conductance catheter measurement in the RV, they found that PI increased with sudden complete occlusion of a branch PA with a large-caliber balloon. Although this study supports the concept that impedance to antegrade arterial flow exacerbates PI, this does not resemble the clinical scenario of chronic branch pulmonary stenosis in the setting of PI. To our knowledge, the current report is the first study directly evaluating the effects of relief of branch PA stenosis on PI.

We have demonstrated that relief of unilateral branch PA stenosis alleviates PI in an animal model. Cardiac MRI was used to detect changes in RV function and volume after the intervention, because MRI has become the gold standard for RV volumetric analysis. ${ }^{25-27}$ In the current study, mean PI decreased from $38.2 \%$ to $27.4 \%$ without any intervention on the pulmonary valve itself. This is significant because intervention on branch PA stenosis may provide an opportunity to reduce PI in this challenging patient population. Further, stenting to relieve LPA stenosis is a durable intervention. Stenting of branch pulmonary stenosis has been performed for approximately two decades. Investigators reporting on the intermediate and long-term results have 
shown that stenting is highly effective at durable resolution of branch PA stenosis. ${ }^{28-31}$ Thus, although relief of LPA stenosis obviously does not abolish PI, its positive benefits in alleviating PI may be long-lasting. Reduction in PI in this study was associated with increased net flow to the left lung and volume unloading of the RPA. The RV systolic function improved, as indicated by increased ejection fractions. Velocity mapping at the aorta demonstrated an improvement in cardiac index after stenting. The results of this study underscore the importance of continued surveillance and treatment of postoperative branch PA stenosis in patients with TOF. Interventional catheterization in this setting serves an important complementary role to surgical repair by mitigating PI.

Studies in humans with PI have demonstrated that pulmonary valve replacement is associated with a decrease in RV EDV and a decrease in RV EDV:LV EDV ratio. In these studies, follow-up imaging obtained at 6 or 12 months after intervention demonstrates lower RV EDV with no change in RV stroke volume or RV ESV. In the current study, although there was a trend toward lower RV EDV, this decrease was not statistically significant. Although there was no significant change in RV EDV:LV EDV ratio, the repeat evaluation in the current study occurred at 1 week after LPA stenting. Short follow-up time may have limited the ability to detect decreases in RV EDV. RV ejection fraction improved from $53 \%$ to $58 \%$ in the short follow-up period. This increased systolic performance of the RV may well reflect the increased cross-sectional diameter available for antegrade flow.

The contribution of PI to RV systolic function is complex and has been incompletely explained. Our study, however, demonstrates that RV systolic function can respond, and improve, quickly after relieving branch pulmonary arterial obstruction and thereby reducing PI.

\section{LIMITATIONS}

This study used an animal model of PI with unilateral branch pulmonary stenosis. This model was not a true anatomic model of repaired TOF. In addition, the number of animals was relatively small. Finally, the follow-up MRI scans were performed only 1 week after intervention because of protocol constraints. Studies evaluating the long-term effects of LPA stenting on PI and ventricular function are warranted. How these findings relate to human patients born with congenital heart disease is uncertain. Corollary human studies measuring PI and RV function before and after intervention on branch PA stenosis are warranted.

\section{CONCLUSIONS}

Relief of unilateral branch PA stenosis reduces PI in this animal model. The improvement in antegrade flow through the MPA after relief of branch stenosis corresponds to improve- ment in RV systolic performance and cardiac index. Stenting offers the potential to alleviate PI in patients with incompetent pulmonary valves and unilateral branch pulmonary stenosis. In patients with PI, stenting should be strongly considered as a means of nonsurgical palliation. The relationship between alleviation of PI and improved ventricular function remains incompletely explained, and further investigation is warranted.

\section{References}

1. Gatzoulis MA, Balaji S, Webber SA, Siu SC, Hokanson JS, Poile C, et al. Risk factors for arrhythmia and sudden cardiac death late after repair of tetralogy of Fallot: a multicentre study. Lancet. 2000;356:975-81.

2. Harrison DA, Harris L, Siu SC, MacLoghlin CJ, Connelly MS, Webb GD, et al. Sustained ventricular tachycardia in adult patients late after repair of tetralogy of Fallot. J Am Coll Cardiol. 1997;30:1368-73

3. Rowe SA, Zahka KG, Manolio TA, Horneffer PJ, Kidd L. Lung function and pulmonary regurgitation limit exercise capacity in postoperative tetralogy of Fallot. J Am Coll Cardiol. 1991;17:461-6.

4. Ghai A, Silversides C, Harris L, Webb GD, Siu SC, Therrien J. Left ventricular dysfunction is a risk factor for sudden cardiac death in adults late after repair of tetralogy of Fallot. J Am Coll Cardiol. 2002;40:1675-80.

5. Wessel HU, Cunningham WJ, Paul MH, Bastanier CK, Muster AJ, Idriss FS. Exercise performance in tetralogy of Fallot after intracardiac repair. $J$ Thorac Cardiovasc Surg. 1980;80:582-93.

6. Davlouros PA, Karatza AA, Gatzoulis MA, Shore DF. Timing and type of surgery for severe pulmonary regurgitation after repair of tetralogy of Fallot. Int J Cardiol. 2004;97(Suppl 1):91-101.

7. Therrien J, Provost Y, Merchant N, Williams W, Colman J, Webb G. Optimal timing for pulmonary valve replacement in adults after tetralogy of Fallot repair. Am J Cardiol. 2005;95:779-82.

8. Therrien J, Siu SC, McLaughlin PR, Liu PP, Williams WG, Webb GD. Pulmonary valve replacement in adults late after repair of tetralogy of Fallot: are we operating too late? J Am Coll Cardiol. 2000;36:1670-5.

9. Bove EL, Kavey RE, Byrum CJ, Sondheimer HM, Blackman MS, Thomas FD. Improved right ventricular function following late pulmonary valve replacement for residual pulmonary insufficiency or stenosis. J Thorac Cardiovasc Surg. 1985;90:50-5.

10. van Huysduynen BH, van SA, Swenne CA, Maan AC, van Eck HJ, Schalij MJ, et al. Reduction of QRS duration after pulmonary valve replacement in adult Fallot patients is related to reduction of right ventricular volume. Eur Heart J. 2005; 26:928-32.

11. Khambadkone S, Coats L, Taylor A, Boudjemline Y, Derrick G, Tsang V, et al. Percutaneous pulmonary valve implantation in humans: results in 59 consecutive patients. Circulation. 2005;112:1189-97.

12. Uretzky G, Puga FJ, Danielson GK, Hagler DJ, McGoon DC. Reoperation after correction of tetralogy of Fallot. Circulation. 1982;66(2 Pt 2):I202-8.

13. McElhinney DB, Parry AJ, Reddy VM, Hanley FL, Stanger P. Left pulmonary artery kinking caused by outflow tract dilatation after transannular patch repair of tetralogy of Fallot. Ann Thorac Surg. 1998;65:1120-6.

14. Sharma SN, Sharma S, Shrivastava S, Rajani M, Tandon R. Pulmonary arterial anatomy in tetralogy of Fallot. Int J Cardiol. 1989;25:33-7.

15. Rhodes J, Dave A, Pulling MC, Geggel RL, Marx GR, Fulton DR, et al. Effect of pulmonary artery stenoses on the cardiopulmonary response to exercise following repair of tetralogy of Fallot. Am J Cardiol. 1998;81:1217-9.

16. Ilbawi MN, Idriss FS, DeLeon SY, Muster AJ, Gidding SS, Berry TE, et al. Factors that exaggerate the deleterious effects of pulmonary insufficiency on the right ventricle after tetralogy repair. Surgical implications. J Thorac Cardiovasc Surg. 1987;93:36-44.

17. Hawk C, Leary S, Morris T. Formulary for Laboratory Animals. 3rd edition. Durham, NC: Blackwell Publishing; 2005.

18. Bouzas B, Kilner PJ, Gatzoulis MA. Pulmonary regurgitation: not a benign lesion. Eur Heart J. 2005;26:433-9.

19. Wu MT, Huang YL, Hsieh KS, Huang JT, Peng NJ, Pan JY, et al. Influence of pulmonary regurgitation inequality on differential perfusion of the lungs in tetralogy of Fallot after repair: a phase-contrast magnetic resonance imaging and perfusion scintigraphy study. J Am Coll Cardiol. 2007;49:1880-6.

20. Hirsch JC, Mosca RS, Bove EL. Complete repair of tetralogy of Fallot in the neonate: results in the modern era. Ann Surg. 2000;232:508-14. 
21. Dowdle SC, Human DG, Mann MD. Pulmonary ventilation and perfusion abnormalities and ventilation perfusion imbalance in children with pulmonary atresia or extreme tetralogy of Fallot. J Nucl Med. 1990;31:1276-9.

22. Bouzas B, Kilner PJ, Gatzoulis MA. Pulmonary regurgitation: not a benign lesion. Eur Heart J. 2005;26:433-9.

23. de Ruijter FT, Weenink I, Hitchcock FJ, Meijboom EJ, Bennink GB. Right ventricular dysfunction and pulmonary valve replacement after correction of tetralogy of Fallot. Ann Thorac Surg. 2002;73:1794-800.

24. Chaturvedi RR, Kilner PJ, White PA, Bishop A, Szwarc R, Redington AN. Increased airway pressure and simulated branch pulmonary artery stenosis increase pulmonary regurgitation after repair of tetralogy of Fallot. Real-time analysis with a conductance catheter technique. Circulation. 1997;95:643-9.

25. Higgins CB, Sakuma H. Heart disease: functional evaluation with MR imaging. Radiology. 1996;199:307-15.

26. Kang IS, Redington AN, Benson LN, Macgowan C, Valsangiacomo ER, Roman K, et al. Differential regurgitation in branch pulmonary arteries after repair of tetralogy of Fallot: a phase-contrast cine magnetic resonance study. Circulation. 2003; 107:2938-43
27. Sorrell VL, Altbach MI, Kudithipudi V, Squire SW, Goldberg SJ, Klewer SE. Cardiac MRI is an important complementary tool to Doppler echocardiography in the management of patients with pulmonary regurgitation. Echocardiography. 2007;24:316-28.

28. O'Laughlin MP, Slack MC, Grifka RG, Perry SB, Lock JE, Mullins CE. Implantation and intermediate-term follow-up of stents in congenital heart disease. Circulation. 1993;88:605-14.

29. Shaffer KM, Mullins CE, Grifka RG, O'Laughlin MP, McMahon W, Ing FF, et al. Intravascular stents in congenital heart disease: short- and long-term results from a large single-center experience. J Am Coll Cardiol. 1998;31:661-7.

30. Spadoni I, Giusti S, Bertolaccini P, Maneschi A, Kraft G, Carminati M. Long-term follow-up of stents implanted to relieve peripheral pulmonary arterial stenosis: hemodynamic findings and results of lung perfusion scanning. Cardiol Young. 1999; 9:585-91.

31. Stanfill R, Nykanen DG, Osorio S, Whalen R, Burke RP, Zahn EM. Stent implantation is effective treatment of vascular stenosis in young infants with congenital heart disease: acute implantation and long-term follow-up results. Catheter Cardiovasc Interv. 2008;71:831-41. 